\title{
A meeting framework for inclusive and sustainable science
}

\author{
The $A B C D$ conference format (All continents, Balanced gender, low Carbon transport, Diverse backgrounds) mixes \\ live-streamed and pre-recorded talks with in-person ones to reflect a diverse range of viewpoints and reduce the \\ environmental footprint of meetings while also lowering barriers to inclusiveness.
}

\author{
Rosetta C. Blackman, Andreas Bruder, Francis J. Burdon, Peter Convey, W. Chris Funk, Sonja C. Jähnig, \\ Mary Alphonce Kishe, Marcelo S. Moretti, Vianny Natugonza, Jan Pawlowski, Rachel Stubbington, \\ Xiaowei Zhang, Ole Seehausen and Florian Altermatt
}

$\mathrm{C}$ onferences are, next to publications in scientific journals, the most commonly used format to present and disseminate recent advances in scientific research. Conferences are also important to synthesize knowledge, especially through sessions dedicated to a specific topic, to bring together different views and to explore solutions to shared problems. A wide range of diverse backgrounds of participants is often seen as a key element to facilitate novel insights, promote unconventional solutions and advance science. However, while there are hundreds of conferences and symposia within individual research fields, the examples of those achieving this seem to be relatively few. Scientific culture can also be inward facing with many symposia drawing on participants repetitively from a small sector of the global community both within and beyond disciplines. Think of your own favourite conference and ask yourself what fraction of global cultures are typically represented among the keynotes, speakers and participants. We think this must change in the interest of both science and society. In the context of major global issues that need to be addressed with urgency, this is truer now than ever before.

An area where inclusiveness and novel synthesis is especially urgent are the sustainability sciences linked to global biodiversity loss and climate change $\mathrm{e}^{1-3}$. It is questionable whether the classical way of organizing scientific conferences is most effective for addressing such complex scientific subjects. This may be especially true for finding solutions to environmental problems that also have a strong social science component. Furthermore, the current way of organizing conferences involves considerable travelling, especially by aeroplanes, which not only results in large and unwanted carbon footprints ${ }^{4}$, but also perpetuates inequalities in participation due to uneven financial constraints.

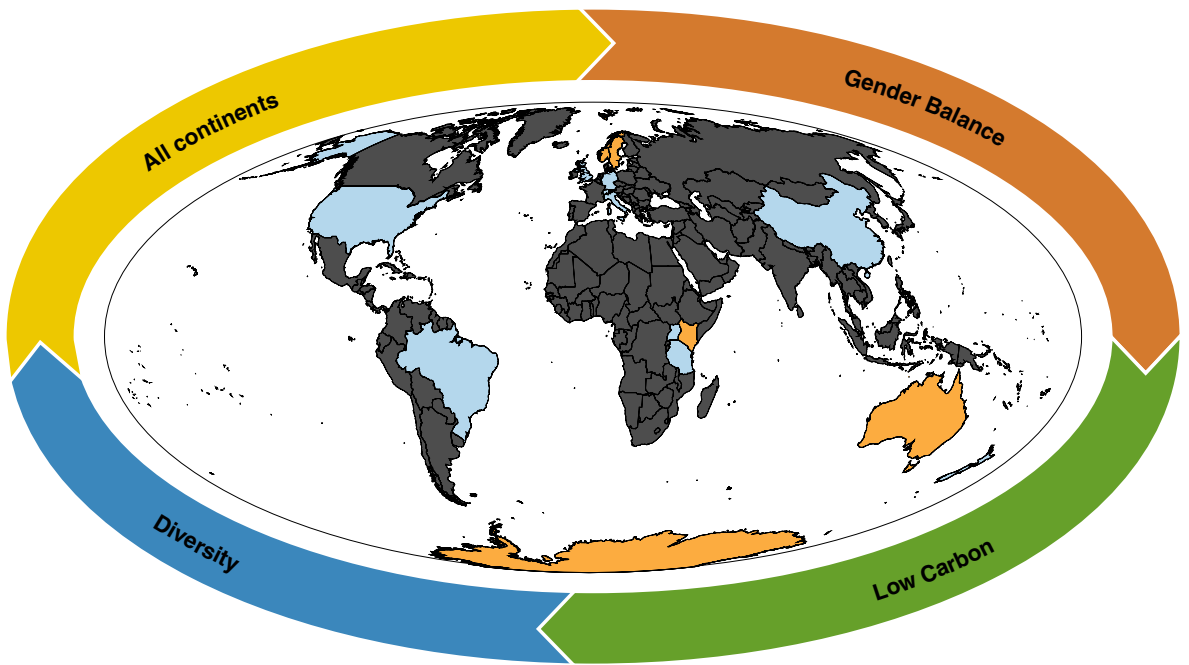

Fig. 1 | The $A B C D$ conference format featuring examples of the World Biodiversity Forum contributors. The $A B C D$ conference format embraces the four criteria 'All continents, gender Balance, low Carbon, and Diverse backgrounds' in order to address inclusivity and combat inequity within the science community, while lowering carbon footprint when attending international conferences. The speakers who contributed to the World Biodiversity Forum Aquatic Biodiversity session following the $A B C D$ framework came from (blue) or discussed research based on (blue and orange) countries and geographic regions across all continents. Publ. note: Springer Nature is neutral about jurisdictional claims in maps.

Here, we propose a new meeting format as a means to achieve greater inclusivity at reduced carbon footprint, and hence greater benefits relative to the aforementioned costs of international conferences. Our model allows a concerted and integrative approach for disciplinary and interdisciplinary conferences to improve sustainability and to close knowledge gaps and bridge barriers to exchange across cultural backgrounds.

\section{The ABCD format}

Scientific evidence shows that diverse and inclusive groups are better at addressing and solving complex problems $s^{5,6}$ such as those faced today in sustainability sciences, particular in the context of biodiversity loss. To better achieve this at scientific conferences, we have developed a framework we term the ABCD conference format (\#ABCD_conference) (Fig. 1). This format is based on the premise that the speakers invited for a symposium are selected, beyond scientific excellence and relevance, based on the following criteria: From All continents, gender Balance, attendance by low Carbon transport, and represent Diverse backgrounds. We briefly explain and justify these four criteria. 


\section{Box 1 | Suggestions to consider when using the ABDC conference format}

The listed suggestions are gathered from both organizers and speakers attending the session 'Aquatic Biodiversity: state and challenges ahead' at the 2020 World Biodiversity Forum:

- Prior to the event, have a virtual 'ice-breaker' with all the speakers to discuss what they will be presenting and how their research may fit together.

- Run through presenting procedures (including technical requirements: microphone and camera) with all speakers using new methods to present (remote or pre-record).

- Ask all presenters to add a photo to all remote or pre-recorded talks on their first slide to make them visible as a person to the audience.

- If facilities allow, have a camera on both the audience and presenter to increase engagement for both parties.

- Ensure pre-recorded presentations are within the time allowance, as time-corrections are not possible.

- Agree on a protocol to ensure remote presenters are aware of the time allowance and if they have exceeded it.

- Local organizers and session chairs may use a flipped classroom for questions - organizers view the pre-recorded talks prior to the session, and bring up discussion points or even questions to the audience, which will then engage in a discussion.

- Engage audience in discussion via Slido.com, Twitter.com or other platforms. Allowing questions to be shared with respective presenters from other time zones when they are available.

- Have a repository where all the talks will be deposited and shared with all those interested.

- Due to the nature of remote and pre-recorded talks, ensure you have sufficient time for discussion, questions and changing of presenters. This may be a bit longer than in classic meeting settings, where there is often a 15-minute slot for a 13-minute talk plus two minutes of questions, directly followed by the next talk. Allow an additional 2-5 minutes for discussion and moving to the next speaker (be it live-streamed, pre-recorded or in person).

- Allow sufficient time for discussion at the end of the session.

\section{All continents: representation and} dissemination of and exchange between research being carried out throughout the world is of great importance. If we do not bring together scientists from all corners and cultures of the globe, we risk missing important links with our own research, failing to recognize ideas developed and insights obtained elsewhere in the world, and wasting precious time and funding on repeating work already done.

Gender Balance: the lack of gender equity in science is widely recognized ${ }^{7}$. Speakers identifying as male, female or non-binary bring different perspectives. However, male speakers often dominate meetings in numbers and functions ${ }^{8}$. Ensuring equal representation and equity of gender broadens the range of perspectives, insights and experiences that are needed to nourish knowledge, and create role models for early-career scientists and future generations ${ }^{9}$.

Low Carbon: carbon emissions created by travelling are a well-founded concern of delegates when attending conferences, especially in the sustainability sciences ${ }^{4}$.
However, alternatives are rarely provided. Unfortunately, low carbon emission requirements can be in direct conflict with the desire to increase efforts for bringing scientists together across continents. In the $\mathrm{ABCD}$ conference format we promote mixing live-streamed and pre-recorded talks by contributors from afar with live in-person talks by contributors from nearby who travel on low carbon emission means. Low-cost and easily accessible technology allows for active engagement without travel, lowering both costs and carbon emissions to attend international conferences. Moreover, rotating the venue for recurrent $\mathrm{ABCD}$ conferences between continents has the potential to overcome the all-apparent inequities between scientists from different continents.

Diversity: ensuring inclusivity of diverse cultural backgrounds within a conference agenda is of utmost importance for several reasons. Here, we define cultural diversity as, but not limited to, ethnicity, religion and language. Cultural diversity and equitable representation at scientific meetings facilitate the exchange of ideas and insights grounded in diverse perspectives. It increases participation, understanding and fosters scientific advance and collaboration ${ }^{6}$ now and even more so in the future through removing inequalities in the visibility of role models.

\section{Case study at the World Biodiversity Forum 2020}

We believe that the ABCD conference format will not only connect people but also create trust within the scientific community, enabling better exchange of ideas and increased impact in working towards solutions, be it the effects of global climate change, regional biodiversity loss, or in any other field of research. We recently tested this format in the session on 'Aquatic Biodiversity: state and challenges ahead', organized and held at the World Biodiversity Forum from 22 to 28 February 2020 in Davos, Switzerland. Prior to the session we gave invited speakers the option of live-streaming, pre-recording or attending via low carbon emission transport to deliver their presentation. On the day, we had presentations in all three formats which gave an overview on the state and threats to aquatic biodiversity, discussed the underlying drivers, and outlined how this affects the ecological and evolutionary functioning of the ecosystems. Here, we give our first-hand experience of the gains but also challenges we experienced from the organizers' and speakers' perspective:

Organizers. As organizers aiming to meet the four criteria set out under the $\mathrm{ABCD}$ format, we quickly realized constraints due to the limited reach of our own professional networks. Institutions in the 'West' with additional strongholds in Asia and only relatively few researchers elsewhere dominate aquatic biodiversity representation. Not only are our own networks highly biased, but we realized that the relatively isolated researchers in the field outside the stronghold regions are not well networked amongst themselves either. We therefore needed to reach out beyond our own established networks and those of our contacts elsewhere, which led to a number of 'dead-ends' with no responses from potential candidates. Our attempts to have gender balance turned out to be especially challenging, and several female speakers invited unfortunately did not respond to our request for unknown reasons. Those who did not respond to our request may not have in part due to our novel request to participate in a session without physical attendance or networking, which is often a key reason to attend conferences. Such constraints may be overcome in the future 
by increased awareness of the aims of the ABCD format and through 'directed growth' of our own professional networks beyond our immediate geographic or scientific vicinity.

\section{Running the session. By using}

live-streaming and pre-recorded talks, all speakers not attending in person were suitably prepared to present on the day. By using these methods, additional consideration needs to be taken for timing, particularly with remote talks running over time. This can be addressed by the addition of web cameras faced at both presenter and audience, enabling the chair to communicate the time allowance. In our session, we promoted discussion via social media and an online conference question platform. However, we got only a few discussion points from this, which may be due to the novel concept and may likely evolve if this method becomes more common. One way to increase discussion could be to make questions of the audience visible to both speaker and audience in a live ticker, as is already possible in some video conferencing programs.

Speakers. Contributors to the session were introduced to the ABCD format and the goals of the session when being invited to participate. This is key and perhaps cannot be overemphasized when organizing a session using the ABCD format. Several participants highlighted the potential for networking and discussion as an area for improvement. This can be addressed with further engagement, such as pre-conference discussion among the speakers, increased engagement with online discussion tools, and an increased familiarity of presenters/ attendees with this new format.

Outcomes. By using the ABCD conference format, the session encompassed a nuanced attitude and recognized some new key areas of research across geography. A number of remote and pre-recorded speakers highlighted that they felt comfortable presenting their research using these methods, and that it enabled contributions and opportunities which would not have existed otherwise. (See Box 1 for further information on $\mathrm{ABCD}$ format planning.)

Our session focused on the state and change of freshwater ecosystems in the context of global change. Rivers and lakes are among the most biodiverse ecosystems worldwide and provide essential resources and services for humans, including drinking water, fisheries, transport or recreation. However, they are also globally among the most threatened ecosystems ${ }^{10}$. A highlight of our session was the identification of overlapping key findings and hitherto missing links between different initiatives that would likely not have emerged at this point in time without the ABCD approach. As an example, the session identified missing links between biodiversity data mobilization initiatives in different parts of the world (for example, the Freshwater Biodiversity Observation Network (FreshwaterBON) having a stronghold in Europe, while JRS Biodiversity Foundation and the Global Biodiversity Information Facility (GBiF) dominate in Africa) and the respective stakeholders and scientists. It also became obvious that finding solutions to the multiple risks threatening freshwater biodiversity ${ }^{11}$ is in large parts of the world challenged by lack of basic data (due to a paucity of species identification experts and funding), lack of access to databases, and lack in common protocols. A discrepancy between limiting factors and necessary solutions was matched by backgrounds of attendees, showing that only an inclusive approach allows discussions about possible ways forward that create impact beyond the regional realm.

\section{Beyond the conference}

The need for new formats for scientific conferences, and scientific meetings in general, has not only been discussed in the context of sustainability, but has increased relevance now due to the ongoing COVID-19 crisis. With regional and global travel restrictions implemented by many institutions and governments in order to mitigate the SARS-CoV-2 pandemic, attendance of scientific meetings is affected $^{12}$. However, at many universities and research institutes this challenge has been met by moving to online teaching and online meetings at very short notice, demonstrating that new formats of interaction and conferencing are realistic and doable.

We conclude, and hope, that the ABCD format should not be restricted to conferences, but can also be applied to working groups and research projects. We envision that a more inclusive and sustainable approach will not only reduce the inequities and carbon footprints, but become essential for the finding of solutions to complex multi-faceted challenges in sustainability sciences. These solutions are indeed urgently needed to find rapid science-based solutions for immediate problems, such as the current COVID19 crisis $^{13}$, but also for more persistent problems, such as to bend the curve in the ongoing biodiversity crisis $^{14}$.

Rosetta C. Blackman (D) 1,2 Andreas Bruder (D) ${ }^{3}$, Francis J. Burdon (D),
Peter Convey (D) 5, W. Chris Funk (D)6, Sonja C. Jähnig (D)7, Mary Alphonce Kishe (D)8, MarceloS.Moretti (D) ${ }^{9}$, Vianny Natugonza (D)10, JanPawlowski (DD)11,12,13, RachelStubbington (DD14, Xiaowei Zhang (D) 15, Ole Seehausen (iD) 16,17,18凶 and Florian Altermatt (iD) 1,2,18

${ }^{1}$ Department of Aquatic Ecology, Eawag, Swiss Federal Institute of Aquatic Science and Technology, Dübendorf, Switzerland. ${ }^{2}$ Department of Evolutionary Biology and Environmental Studies, University of Zurich, Zurich, Switzerland. ${ }^{3}$ Laboratory of Applied Microbiology, University of Applied Sciences and Arts of Southern Switzerland, Bellinzona, Switzerland. ${ }^{4}$ Department of Aquatic Sciences and Assessment, Swedish University of Agricultural Sciences, Uppsala, Sweden. ${ }^{5}$ British Antarctic Survey, NERC, Cambridge, UK. ${ }^{6}$ Department of Biology, Graduate Degree Program in Ecology, Colorado State University, Fort Collins, CO, USA. ${ }^{7}$ Leibniz-Institute of Freshwater Ecology and Inland Fisheries (IGB), Department of Ecosystem Research, Berlin, Germany. ${ }^{8}$ Tanzania Fisheries Research Institute (TAFIRI), Dar es Salaam, Tanzania. ${ }^{9}$ Laboratory of Aquatic Insect Ecology, Universidade Vila Velha, Vila Velha, Brazil. ${ }^{10}$ National Fisheries Resources Research Institute (NaFIRRI), Jinja, Uganda. ${ }^{11}$ Department of Genetics and Evolution, University of Geneva, Geneva, Switzerland. ${ }^{12}$ Institute of Oceanology, Polish Academy of Sciences, Sopot, Poland. ${ }^{13} I D-G e n e$ ecodiagnostics, Campus Biotech Innovation Park, Geneva, Switzerland. ${ }^{14}$ School of Science and Technology, Nottingham Trent University, Nottingham, UK. ${ }^{15}$ School of the Environment, Nanjing University, Nanjing, China. ${ }^{16}$ Department of Fish Ecology \& Evolution, Eawag, Swiss Federal Institute of Aquatic Science and Technology, Kastanienbaum, Switzerland. ${ }^{17}$ Division of Aquatic Ecology \& Evolution, Institute of Ecology \& Evolution, University of Bern, Bern, Switzerland. ${ }^{18}$ These authors contributed equally: Ole Seehausen, Florian Altermatt.

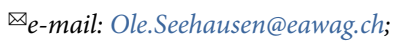

Florian.Altermatt@ieu.uzh.ch

Published online: 24 April 2020

https://doi.org/10.1038/s41559-020-1190-x

References

1. IPCC Special Report on Global Warming of $1.5^{\circ} \mathrm{C}$ (eds Masson-Delmotte, V. et al.) (WMO, 2018).

2. Special Report on Biodiversity and Ecosystem Services (eds Díaz, S., Settele, J. \& Brondizio, E.) (IPBES, 2019).

3. Abiven, S. et al. Curr. Opin. Environ. Sustain. 29, 215-222 (2018).

4. Abbott, A. Nature 577, 13 (2020).

5. Adams, J. Nature 497, 557-560 (2013).

6. Freeman, R. B. \& Huang, W. Nature 513, 305 (2014).

7. Shannon, G. et al. Lancet 393, 560-569 (2019).

8. Farr, C. M. et al. BioScience 67, 464-468 (2017).

9. Campbell, L. G., Mehtani, S., Dozier, M. E. \& Rinehart, J. PLoS ONE 8, e79147 (2013).

10. Reid, A. J. et al. Biol. Rev. 94, 849-873 (2019).

11. Dudgeon, D. Curr. Biol. 29, 960-967 (2019).

12. Viglione, G. Nature 579, 327-328 (2020).

13. Li, R. et al. Science https://doi.org/10.1126/science.abb3221 (2020).

14. Tickner, D. et al. BioScience 70, 330-342 (2020). 
Acknowledgements

We thank the University of Zurich Research Priority

Programme on Global Change and Biodiversity

(URPP GCB) for organizing the World Biodiversity

Forum and hosting our session, as well as all attendees of

the session for their comments, contributions and openness

to this new format. Funding is from the University of

Zurich URPP GCB and the Swiss National Science

Foundation Grant No. 31003A_173074 to F.A. and
Swiss National Science Foundation Grant No. CRSII5_183566 / 1 to O.S.

Author contributions

R.C.B., O.S. and F.A. developed the ABCD format,

organized, and hosted a session using the format

at the World Biodiversity Forum with R.C.B., A.B.,

F.J.B., P.C., W.C.F., S.C.J., M.A.K., M.S.M., V.N., J.P., R.S

and X.Z. as speakers. R.C.B., O.S. and F.A. wrote the first draft of the manuscript, and A.B., F.J.B., P.C., W.C.F., S.C.J., M.A.K., M.S.M., V.N., J.P., R.S. and X.Z. contributed to it. R.C.B., O.S. and F.A. wrote the final version of the manuscript. All authors approved this final version. R.C.B. produced the figure.

Competing interests

The authors declare no competing interests. 